Mitteilungen der Österreichischen Geographischen Gesellschaft, 160. Jg., S. 392-397

(Annals of the Austrian Geographical Society, Vol. 160, pp. 392-397)

Wien (Vienna) 2018, https://doi.org/10.1553/moegg160s392

\title{
Christian Vielhaber zum 70. Geburtstag
}

\author{
Christiane HintermanN und Herbert PICHLER, Wien*
}

mit 1 Abb. im Text

Über Christian Vielhaber gibt es unzählige Geschichten zu erzählen, die meisten können wir hier nicht veröffentlichen.

Und das spricht für Christian Vielhaber.

Christian VIELHABER anlässlich seines 70. Geburtstages zu würdigen, ist eine willkommene Gelegenheit, einem Mentor, Wegbegleiter und Freund Dank zu sagen und sich gemeinsam öffentlich zu erinnern. Wohl wissend, dass er traditionelle akademische Rituale der Würdigung für sich selbst als wenig bedeutsam erachtet und bereits etwas würdigungsmüde wirkt. Kein Wunder, wer kann schon von sich behaupten, Zeit seines aktiven universitären Wirkens zwei Festschriften gewidmet bekommen zu haben. Zwei Festschriften, die jeweils eigeninitiativ und selbstorganisiert von einer Gruppe ehemaliger Studierender und Wegbegleiter/innen verfasst wurden. Bereits zu seinem „Fünfziger" wurde er mit „Geografie, Wirtschaftskunde und andere Ungereimtheiten“ (GoETZ et al. 1998) überrascht, zehn Jahre später schaffte es die Festschrift „kind : macht : raum“ (DoBLER et al. 2008),

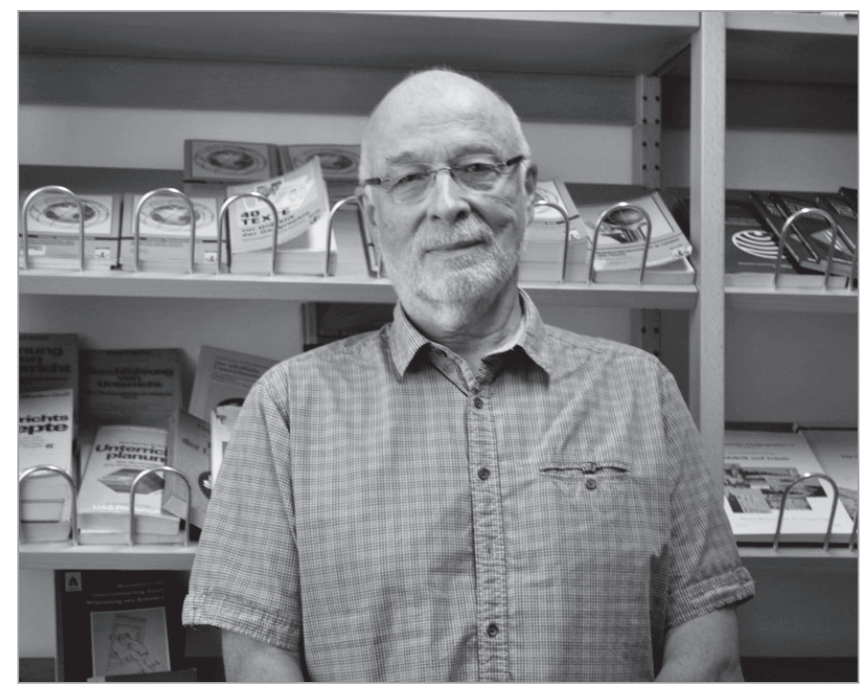

Christian VIELHABER

\footnotetext{
* Mag. Dr. Christiane Hintermann, Mag. Herbert Pichler, beide: Universität Wien, Institut für Geographie und Regionalforschung, Arbeitsgruppe Fachdidaktik Geographie und wirtschaftliche Bildung, Universitätsstraße 7, A-1010 Wien; E-Mail: christiane.hintermann@univie.ac.at; herbert.pichler@univie.ac.at
} 
den nicht eingeweihten Jubilar zu erfreuen und sichtlich stolz zu machen. Schließlich wurde sein Übertritt in den Unruhestand im Rahmen der österreichweiten Tagung „Zukunft Fachdidaktik GW“ in Schlierbach 2016 nachträglich gefeiert: Der Vorwand eines Kamingesprächs 4.0 vor virtuellem Kaminknistern über Konstruktivismus und Kontingenz erlaubte uns, Christian ein drittes Mal zu überraschen. Das freundschaftlich-kollegiale Streitgespräch mit Tilman RHODE-JÜCHTERN mündete in eine pointierte Laudatio (vgl. PICHLER und KesSel 2016, S. 54f). Womit uns das beinahe Unmögliche gelang: Dem beredten langjährigen Sprachrohr der österreichischen Fachdidaktik GW fehlten für einen kurzen Moment die Worte.

Den nun folgenden Einblicken in den beruflichen Werdegang und das vielfältige bildungs- und gesellschaftspolitische Wirken werden kurze prägnante Äußerungen und Zitate von Christian Vielhaber vorangestellt, die zentrale Positionierungen zum Ausdruck bringen.

\section{„Schule muss anders gehen!“ - Der Lernende, Kollege und Mitstreiter}

In gewisser Weise ist es einem glücklichen Zufall zu verdanken, dass Christian VIELHABER zur Geographie und damit zur Fachdidaktik Geographie und Wirtschaftskunde gefunden hat, denn nach seiner Matura am Bundesgymnasium Billrothstraße in Wien $19 \mathrm{im}$ student/innen/bewegten Jahr 1968 drängte es den Studienbeginner zunächst in eine andere Richtung. Von seinem Wunschstudium der Publizistik schreckten ihn bei der Immatrikulation die „Studierendenmassen“ ab und so inskribierte er die Lehramtsfächer Geographie und Wirtschaftskunde sowie Anglistik und Amerikanistik. Bereits während des Studiums, ab 1973, begann seine Mitwirkung an der universitären Forschung und Lehre. Gemeinsam mit Karl Husa bekleidete er die Agenden einer wissenschaftlichen Hilfskraft bei Univ.-Prof Dr. Ernest Troger, der die Professur für Länderkunde und Allgemeine Geographie innehatte. Ab 1975 wurde er nach seinem Studienabschluss zum Universitätsassistenten am gleichen Ordinariat bestellt. Neben seinen universitären Verpflichtungen sammelte Christian VIELHABER von 1973 bis 1976 Praxiserfahrung als Vertragslehrer in zwei Wiener Gymnasien (BRG 19 und BRG 8), die sein Interesse an der Weiterentwicklung der Fachdidaktik GW vertiefte.

Die wichtigen Qualifikationsarbeiten, die Dissertation 1983 sowie die Habilitation 1988 samt damit verbundener Venia Legendi für Humangeographie und Fachdidaktik Geographie und Wirtschaftskunde sollen im folgenden Abschnitt gewürdigt werden. Sie waren die formalen Voraussetzungen für seine weitere akademische Karriere als Senior Universitätsassistent (1985-1988), als Assistenz-Professor (1989-1996) und schließlich als Außerordentlicher Universitätsprofessor (ab 1996), jeweils am Institut für Geographie der Universität Wien. Als Musterbeispiel für kollegiale Unterstützung ohne Konkurrenzverhältnis soll das so bezeichnete „Triumvirat“ (Karl HusA, Christian Vielhaber und Helmut WoHLSCHLÄGL, einst auch als „die Troger-Buben“ bezeichnet) nicht unerwähnt bleiben, das Christian VielHabers Karriereweg begleitete.

Gelernt hat Christian VIELHABER auch aus unzähligen persönlichen Begegnungen mit Persönlichkeiten, die sein humanistisches und systemkritisches Denken und Handeln befeuert haben. Nur wenige herausragende Impulsgeber können in diesem Rahmen erwähnt werden. In besonderer Weise inspirierte ihn das pionierhafte Wirken des Lehrers, Lehrerausbildners, Lehrplan- und Schulbuchautors Wolfgang SiTTE, dem er im Rahmen der damals einzigen fachdidaktischen Lehrveranstaltung „Besondere Unterrichtslehre (Didaktik und Methodik)“ bereits im Studium am damaligen Geographischen Institut begegnete. Der von Wolfgang SiтTE maßgeblich mitinitiierte zukunftsorientierte Paradigmenwechsel befreite das Schulfach ab den 1970er Jahren von Wissensmast, Stoffhuberei und Länderkunde. Die Geographie wurde um die Wirtschaftskunde erweitert, der Fokus im Sinn der Curricularen Didaktik auf die Bewältigung von Lebenssituationen von Schülerinnen und Schülern neu ausgerichtet (vgl. SitTE und WoHLSCHLÄGL 2001). 
Als Weggefährt/inn/en und Mitstreiter/inn/en von Wolfgang Sitte sind neben Christian VielHABer auch Helmut Wohlschlägl, Maria Hofmann-Schneller, Franz Graf u.a. zu nennen. Den gemeinsamen Anstrengungen um eine Neuausrichtung der Lehramtsausbildung, die Etablierung einer wissenschaftlichen Fachdidaktik Geographie und Wirtschaftskunde sowie eine Professionalisierung der Unterrichtspraxis an den Schulen entsprangen die sukzessive Ausweitung der Fachdidaktik GW in den Lehramts-Curricula und die Entwicklung völlig neuartiger und bis heute einzigartiger Lehrveranstaltungskonzepte, wie die Verzahnung von Unterrichtsplanung, fachbezogenem Schulpraktikum und fachdidaktischem Begleitseminar oder die Universitär-Schulischen Kooperationsprojekte (vgl. Vielhaber 1998). Über die Fachgrenzen hinaus prägend war für Christian VielHaber auch die Zusammenarbeit mit Pädagogin und Psychotherapeutin Gertraud Diem-Wille, mit der er im Rahmen des Modellversuchs „Pädagogikum Geographie“ zusammenarbeitete.

Weitere wichtige Einflüsse auf die Weiterentwicklung der Fachdidaktik GW konnte Christian VIELHABER aus dem engen internationalen Austausch und den Freundschaften mit Größen der deutschsprachigen Geographiedidaktik wie Hermann Schrand, Wulf-Dieter Schmidt-WulfFen, Tilman RHODE-JÜCHTERN und Wolfgang SCHRAMKE gewinnen. Damit positionierte er sich auch international in einem streitbaren Netzwerk, das für eine Didaktik abseits des bewahrenden, funktionalistischen bis utilitaristischen Mainstreams eintritt. Wie sehr seine Arbeit und seine Qualifikationen auch über die Grenzen Österreichs hinaus geschätzt wurden, zeigt sich $u$. a. in einem Ruf auf den Lehrstuhl für Didaktik der Geographie an die Universität Jena 1999, dem er aus privaten Gründen nicht nachkam.

Das gesellschaftspolitische Engagement Christian ViELHABERS für die Kinderrechte, eine gewaltfreie Erziehung und eine humane Schule wurzelt ursächlich in Ohnmachtserfahrungen gegenüber institutioneller schulischer Gewalt während der eigenen Schulzeit im Bundesgymnasium Eisenstadt. Später sensibilisierten ihn die Erfahrungen als Lehrer und Lehrerausbildner genauso wie unzählige direkte Interventionen im Interesse von Schülerinnen und Schülern, denen die Institution Schule macht- und gewaltvoll entgegengetreten war. Die Begegnung mit dem Kinderarzt und Sozialmediziner Hans Czermak inspirierte Christian VielHaber, dieses persönliche Engagement auf eine breitere Basis zu stellen. Er trat dem von Czermak ins Leben gerufenen „Verein zur gewaltfreien Erziehung / Österreichischer Kinderschutzbund“ bei, war dort viele Jahre im Vereinsvorstand aktiv und verbreitet seither bei Tagungen und Vorträgen engagiert und authentisch die Ziele und Forderungen einer gewaltfreien Erziehung. Auch in dieser Tätigkeit spannt er einen Bogen zur Geographie, wenn er zum Beispiel die gewaltvolle Missachtung räumlicher Nutzungsansprüche von Kindern und Jugendlichen thematisiert.

Das mit Unterstützung des damaligen Institutsvorstands, Helmut WoHLSCHLÄGL, 2009 neu errichtete „Fachdidaktikzentrum Geographie und Wirtschaftskunde“ am Institut für Geographie und Regionalforschung der Universität Wien, das Christian VielHaber bis zu seinem Ruhestand im Jahr 2013 leitete, baute er gemeinsam mit Herbert PichleR und Heidrun EdLINGER zu einer niederschwelligen Anlaufstelle für Schülerinnen und Schüler, Studierende, Lehrpersonen, Lektor/inn/ en und alle an Fachdidaktik Interessierten aus. Gemeinsam neu entwickelte Formate wie die „FDZNight" mobilisierten bis zu 400 Besucherinnen und Besucher, darunter viele Absolventinnen und Absolventen. Das ist nur ein Hinweis darauf, dass es Christian VIELHABer immer gelungen ist, ein Netzwerk an engagierten Lehrerinnen und Lehrern an die Universität zu binden, was für die fachdidaktische und schulpraktische Lehre und Ausbildung von großem Wert ist.

\section{„Fachdidaktik ist eine Sozialwissenschaft!“ - Der Forscher, Publizist und Lehrer}

Fachdidaktiker und gleichzeitig Humangeograph (und vice versa) zu sein, hat sich für Christian VielHaber nie ausgeschlossen. Dies zeigt sich in seinen Forschungsarbeiten, Publikationen und 
Schwerpunkten in der Lehre. Als Sozialwissenschaftler mit einer starken Verankerung in der Kritischen Theorie sind es gesellschaftliche Probleme und Veränderungen, die seine wissenschaftliche Aufmerksamkeit auf sich ziehen, sei es im Bereich der Freizeit- und Tourismusforschung, in Bezug auf Bildung und Schule oder wie jüngst bei Fragen der ,retirement migration“. In seiner 1983 abgeschlossenen, mehr als 500 Seiten starken humangeographischen Dissertation „Freizeitwohnsitze in einem großstädtischen Naherholungsraum. Ein sozialgeographischer Beitrag zur Erforschung des Phänomens Freizeitwohnen am Beispiel des Neusiedlerseeraumes“" analysiert er, auf Basis einer umfangreichen Fragebogenerhebung, Freizeitwohnen als eine besonders raumwirksame Form des Freizeitverhaltens. Inwiefern sich die wissenschaftliche Auseinandersetzung mit dem Thema auf sein eigenes „Freizeitwohnsitzverhalten“ ausgewirkt hat, kann hier nicht weiter ausgeführt werden. Dass er einen Teil seiner scheinbar unerschöpflichen Energie im Wald rund um Thal bei Aflenz in der Obersteiermark beim „Schwammerl suchen“ tankt, ist aber zumindest eine begründbare Hypothese.

Ausgehend von der Beschäftigung mit Zweit- und Freizeitwohnsitzen gilt Christian VIELHABERS fachwissenschaftliches Interesse der räumlichen Mobilität von Menschen in ihren verschiedenen Ausdrucksformen, von touristischen Reisen und deren Konsequenzen bis hin zu Fragen der Migration und den sich daraus ergebenden demographischen und gesellschaftlichen Veränderungen. Darüber hinaus beschäftigen ihn - es versteht sich nahezu von selbst - die Lebenswelten von Kindern und Jugendlichen, vor allem im großstädtischen Raum. Sein regionaler Schwerpunkt liegt zum einen in Österreich, und hier vor allem in den nördlichen, östlichen und (süd)östlichen Grenzgebieten. Legionen von Studierenden wurden bei unzähligen Inlandsexkursionen unter der Leitung von Karl Husa und Christian VielHaber mit den Herausforderungen dieser Gebiete und den dort beobachtbaren Phänomenen der „kumulativen Schrumpfung“ oder der „dezentralen Konzentration“ vertraut gemacht. Außerhalb Österreichs und Europas sind es die Länder Südostasiens, die Christian VIELHABER besonders interessieren und die er gemeinsam mit Karl HuSA im Rahmen von dutzenden Auslandsexkursionen und Forschungsaufenthalten bereist hat. Hier sind es vor allem (massen)touristische Phänomene, die seinen Forschergeist anregen. In jüngster Zeit und wieder im Tandem mit seinem Freund und Kollegen Karl HusA rückte die Frage der „retirement migration“ nach Thailand in den Forschungsfokus der beiden „Unruheständler“.

Seine besondere Leidenschaft gilt jedoch der Weiterentwicklung der Fachdidaktik Geographie und Wirtschaftskunde und damit auch des Unterrichtsfaches. Bereits fünf Jahre nach dem Abschluss der Dissertation war seine Habilitation „Perspektiven einer kritisch-pragmatischen Fachdidaktik Geographie" fertig, die das Fundament seiner weiteren Entwicklungsarbeit darstellt. (Zukünftigen) Lehrpersonen Begründungsfähigkeit für die in ihrem $(\mathrm{GW})$ Unterricht nötigen fachlichen, fachdidaktischen und methodischen Entscheidungen zu vermitteln, ist sein zentrales Ziel. Eine Begründungsfähigkeit, die notwendigerweise auf ausgewählten Theorien beruhen muss, treibt ihn bis heute um. Mit den von ihm in die Fachdidaktik eingeführten Vermittlungsinteressen (vgl. VIELHABER 2000) hat er ein Modell zur Planung und Analyse von Unterricht in die fachdidaktische Diskussion eingebracht, das Studierende und Lehrpersonen dabei unterstützt, Unterricht zu strukturieren und sich Klarheit über (vielfach implizite) Lernziele und Interessen zu verschaffen und deren Relevanz zu überprüfen.

Neben seinem umfangreichen Werk an wissenschaftlichen Veröffentlichungen - mehr als 150 fachdidaktische, schulpraktische, bildungspolitische und fachwissenschaftliche Beiträge in Zeitschriften und Sammelbänden - ist der Name Christian VielHaber untrennbar mit der Herausgabe von zwei Publikationsorganen verbunden, die die österreichische und deutschsprachige Geographiedidaktik nachhaltig beeinflusst haben. Zwischen 1988 und 2012 war Christian VIELHABER gemeinsam mit Helmut WoHLSCHLÄGL verantwortlicher Herausgeber der Reihe „Materialien zur Didaktik der Geographie und Wirtschaftskunde“ am Institut für Geographie und Regionalforschung der Universität Wien. Insgesamt 25 Bände zu aktuellen und/oder kontroversen Entwicklungen in der 
Fachdidaktik, alternativen Zugängen und Konzepten, aber auch zu unterrichtspraktischen Beispielen, wurden in diesem Zeitraum veröffentlicht. Daneben war er von 1995 (Heft 57) bis 2010 (Heft 116) Redaktionsmitglied und ein bedeutender Ideenlieferant und Motor der Fachdidaktikzeitschrift „GW-Unterricht“, dem unabhängigen Publikationsorgan für Fachwissenschaft, Fachdidaktik und Schulpraxis GW in Österreich.

Schließlich sind seine Beiträge zu Organisation und Durchführung der „Gesamtösterreichischen Fachdidaktiktagung Geographie und Wirtschaftskunde“ in Haimingerberg ab 1995 erwähnenswert. Sowohl die Zeitschrift GW-Unterricht als auch die Fachdidaktiktagungen in Haimingerberg können als wesentliche Basis für den österreichweiten Austausch und die Vernetzung der Fachdidaktiken an Pädagogischen Hochschulen und Universitäten gelten. Dem Zweck der Vernetzung dienten auch die Fachdidaktiktage im Rahmen der IMST („Innovationen machen Schulen top“) -Tagungen in Klagenfurt, die er über viele Jahre für die Fachgruppe GW organisierte und leitete. Zudem ist Christian Vielhaber ein pointierter kritischer Kommentator bildungspolitischer und fachpolitischer Entwicklungen.

\section{„Was kann ich Gutes für dich tun?“ - Der Mentor, Unterstützer und Ermöglicher}

Wenn man das digitale Vorlesungsverzeichnis der Universität Wien öffnet, würde man nicht glauben, dass sich Christian VIeLHABER seit geraumer Zeit im Ruhestand befindet. Tatsächlich ist er nach wie vor österreichweit und nicht nur an der Universität Wien stark in der Lehre involviert und betreut mehr Abschlussarbeiten als manche aktive Kolleginnen und Kollegen. Er ist ein begeisterter und begeisternder Vortragender und Lehrer. Ihn als Lehrenden zu erleben, bedeutet/e für viele Studierende ein Aha-Erlebnis, mit Stolpersteinen für Denkroutinen und Denkanstößen konfrontiert zu werden, weil er grundsätzliche Fragen aufwirft und diskutiert, die keine (zukünftige) Lehrkraft unberührt lassen, weil er darauf besteht, dass unterrichtliches Handeln ohne theoretische Fundierung beliebig und verzichtbar ist, und weil er seinen Studierenden unendlich viel Zeit schenkt für Rückmeldungen und Diskussionen. So können Texte, die ihm zur Korrektur abgegeben werden, hinter Kommentaren und vorgeschlagenen Änderungen fast nicht mehr sichtbar erscheinen. Auf den ersten Blick löst das keine Begeisterung aus, der zweite Blick eröffnet jedoch, wie sehr die genauen Fragen und Anmerkungen den Text weiter entwickeln helfen. Es ist letztlich ein Ausdruck der Zuwendung und des Interesses für Studierende und Kolleg/inn/en, die sich mit ihm auseinandersetzen. Jeder, der Christian VIelHABER in seinem Arbeitszimmer aufsucht/e, kennt die bei ihm obligatorische einladende Eröffnungsfrage: „Was kann ich Gutes für dich tun?“ Bei ihm war sie nie eine Phrase. Was viele Studierende an ihm schätzen, ist seine hemdsärmelige Interaktion, die Begegnung auf Augenhöhe. Das Duzen ist für ihn Programm, Autoritätsverlust nicht zu befürchten.

Im Gespräch äußert sich Christian VIELHABER nicht selten selbstkritisch über die Nachhaltigkeit des Engagements, über Erfolge und Misserfolge in seinem Einflussbereich, etwa im Bereich der Qualität von Schulbüchern, der Unterrichtsqualität oder im Kontext seines Lieblingsfeindbildes der letzten Jahre: der Kompetenzorientierung. Ob der Beharrungskräfte im Bildungssystem oder der Entdeckung der Langsamkeit von Schulentwicklung mögen einen schon manchmal Selbstzweifel an der Wirksamkeit des eigenen Tuns befallen. Doch Christian VielHaber kann nicht übersehen, dass die österreichische Fachdidaktikszene in Geographie und Wirtschaftskunde kooperiert und sich prächtig entwickelt. Am Relaunch der gesamtösterreichischen Tagung „Zukunft Fachdidaktik GW“ im Jahr 2016 wirkte er selbst mit, der Fachdidaktiktag im Rahmen der IMST-Tagung ist nach wie vor ein lebendiges Austauschforum, die Nachfolgegeneration entwickelt die Fachzeitschrift „GW-Unterricht“ auf der Basis einer institutionenübergreifenden österreichweiten Redaktion in Richtung Professionalisierung weiter (vgl. GW-Unterricht, Nr. 150). 
Wir wollen stellvertretend für die Unzahl an nachhaltigen Impulsen in der Fachdidaktik und für die Schulpraxis, die Christian Vielhaber gegeben hat, eine Frage an den Schluss dieses Beitrags stellen, mit der er viele Lehrpersonen zum Nachdenken und Umdenken bewegen konnte: „Was hast du getan, um dieses ,Nicht genügend“ zu verhindern?“'Auch weil diese Frage eine Haltung ausdrückt, die die Schülerinnen und Schüler in das Zentrum fachdidaktischen Denkens rückt und Lehrpersonen in die Verantwortung nimmt. Was du getan hast, lieber Christian, um die Fachdidaktik GW zu bewegen und Impulse zu setzen, kann diese Würdigung alleine nicht ausdrücken.

\section{Literaturverweise im Text}

Dobler K., Jekel T., Pichler H. (Hrsg.) (2008): kind : macht : raum [Festschrift für Christian VielHaber zum 60. Geburtstag]. Berlin, Wichmann Verlag.

Goetz K., Heintel M., Kana R. (Hrsg.) (1998): Geografie, Wirtschaftskunde und andere Ungereimtheiten. Festschrift für Christian Vielhaber. Wien, WUV Universitätsverlag.

Pichler H., Kessel V. (2016): Tagungsbericht: (Schöne) Neue Arbeitswelten - zwischen New Economy und Prekarisierung. In: GW-Unterricht, 144 (4/2016), S. 50-56.

Rhode-JÜCHTERN T. (2019): Laudatio für Christian Vielhaber. Fabelhafte Einstiege zum Ausstieg von der Uni. Die Chance der Kontingenz. Geographische Erfahrung im Zweiten Blick. In: GW-Unterricht, 153, S. 41-44.

Sitte Ch., Wohlschlägl H. (Hrsg.) (2001): Beiträge zur Didaktik des „Geographie und Wirtschaftskunde“-Unterrichts. Wien, Institut für Geographie und Regionalforschung der Universität Wien (= Materialien zur Didaktik der Geographie und Wirtschaftskunde, 16).

VielHABER Ch. (1998): Universitär-Schulische Kooperationsprojekte. Ein fachdidaktisches und schulpraktisches Ausbildungsangebot am Institut für Geographie und Regionalforschung der Universität Wien. In: Diem-Wille G., Thonhauser J. (Hrsg.): Innovationen in der schulischen Lehrerbildung, Innsbruck, StudienVerlag, S.199-216.

VielHaber Ch. (2000): Vermittlung und Interesse. Zwei Schlüsselkategorien fachdidaktischer Grundlagen im Geographieunterricht. In: VIELHABER Ch. (Hrsg.): Geographiedidaktik kreuz und quer. Vom Vermittlungsinteresse bis zum Methodenstreit - Von der Spurensuche bis zum Raumverzicht. Wien, Institut für Geographie und Regionalforschung der Universität Wien, S. 9 - 26 (= Materialien zur Didaktik der Geographie und Wirtschaftskunde, 15). 